\title{
Rapid reversion of spur cell anemia after liver transplantation
}

\author{
Francesco Santopaolo ${ }^{1,2 *}$, Benedetto $\mathrm{Neri}^{2}$, Alessandra Bosa ${ }^{2}$, Gemma Viola $^{3}$, Ilaria Lenci ${ }^{2}$ and Mario Angelico ${ }^{2}$ \\ ${ }^{1}$ Hepatology Unit, Department of Experimental Medicine and Surgery, University of Rome Tor Vergata, Rome, Italy \\ ${ }^{2}$ Hepatology Unit, Department of Experimental Medicine and Surgery, University of Rome Tor Vergata, Rome, Italy \\ ${ }^{3}$ Department of Internal Medicine, University of Rome Tor Vergata, Rome, Italy
}

\section{Abbreviations: SCA: Spur cell anemia; LT: Liver transplant \\ Introduction}

Spur cell anemia (SCA) is a form of hemolytic anemia historically associated with alcoholic liver cirrhosis. The name of the disease comes from the spike-like projections on the erythrocyte membrane. These are due to a disorder in the cholesterol metabolism causing increased cholesterol to protein and cholesterol to phospholipid ratios in the red cell membrane, making the cells more prone to trapping and destruction by the spleen [1]. One of the first cases of SCA associated to post-alcoholic liver cirrhosis was reported in the New England Journal of Medicine in 1964 [2]. Several investigators have reported the association between alcoholism and SCA, but recent reports demonstrated that the entity might be present in liver disease regardless of etiology. It was reported that the comparison of spur cell rate in patients with alcoholic cirrhosis versus remaining etiologies did not exhibit any difference [3]. The presence of SCA in liver cirrhosis has been associated with advanced disease and poor prognosis [4]. Spur-cell morphological abnormality is reversible in a non-cirrhotic microenvironment and it vanishes after liver transplantation [5]. In the following case report we discuss the history of a patient diagnosed with SCA who, after liver transplantation, promptly resolved the disease.

\section{Clinical Case}

A 59-year-old man, candidate for liver transplantation, with a history of a post-alcoholic liver cirrhosis and hepatocellular carcinoma, was admitted to the emergency department for fever and dyspnoea. Chest X-ray showed abundant right pleural effusion. His hemoglobin level was $6.1 \mathrm{~g}$ per deciliter and additional laboratory tests revealed an elevated indirect bilirubin level of $4 \mathrm{mg}$ per deciliter, an elevated lactate dehydrogenase level and an undetectable haptoglobin level. At admission his MELD score was 24 and his Child Pugh's class was C 12. No evidence of bleeding was found at rectal exam. A peripheral-blood smear showed numerous acanthocytes and the diagnosis of SCA was made. Thoracentesis was performed and the characteristics of pleural fluid were consistent with hepatic hydrothorax, cell count of pleural fluid was $<250$ polymorphonuclear cells for $\mathrm{mm}^{3}$. An evaluation for infection revealed positives blood cultures for Streptococcus Gordonii and therapy with targeted antibiotic was started. He remained dependent on blood transfusions and two weeks later he underwent liver transplantation (LT). One day after LT level of indirect bilirubin decreased from $4 \mathrm{mg}$ per deciliter to $1.4 \mathrm{mg}$ per deciliter with normalization of haptoglobin and LDH level and one week later a peripheral-blood smear showed no acanthocytes. Three months after LT his conditions were excellent and SCA didn't recur.

\section{Discussion}

SCA is an insufficiently investigated entity and its impact on natural history of cirrhosis is yet unclear. There are few reports on its prevalence and survival rate. Sousa el al. examined 339 patients with cirrhosis and found a prevalence of SCA of $4.13 \%$. All the patients were in stage $\mathrm{C}$ of Child Pugh and the presence of spur cells were associated with poor prognosis with a survival of 1 month without LT [3] (Figure 1). However the prevalence of SCA is probably underestimated; SCA, indeed, was found in $31 \%$ of hospitalized cirrhotic patients and the presence of spur cell rate higher or equal to $5 \%$ is a strong independent predictor of mortality [1]. The prevalence of SCA is unknow in patients awaiting for LT and we believe merits further investigation being an additional prognostic indicator in this field. Indeed, taking into account the poor prognosis, it's possible that higher priority for LT should be given to these patients. Completely resolution of SCA has been reported after LT but its impact on peri and post LT survival is

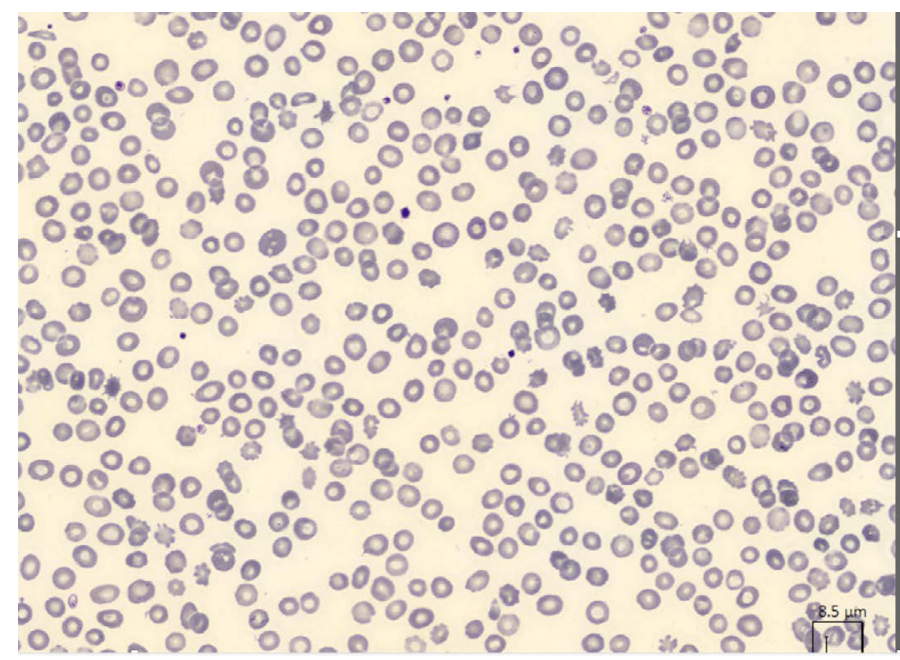

Figure 1. Peripheral blood smear (Hematoxilin-Eosin) showing numerous $(>5 \%)$ spur cells, erythrocytes with irregular surfaces with spike-like projections

${ }^{*}$ Correspondence to: Francesco Santopaolo, Hepatology Unit, Department of Experimental Medicine and Surgery, University of Rome Tor Vergata, Rome, Italy, E-mail: santopaolofrancesco@gmail.com

Key words: Cirrhosis, anemia, liver transplant, acanthocytes

Received: February 03, 2019; Accepted: February 11, 2019; Published: February 15, 2019 


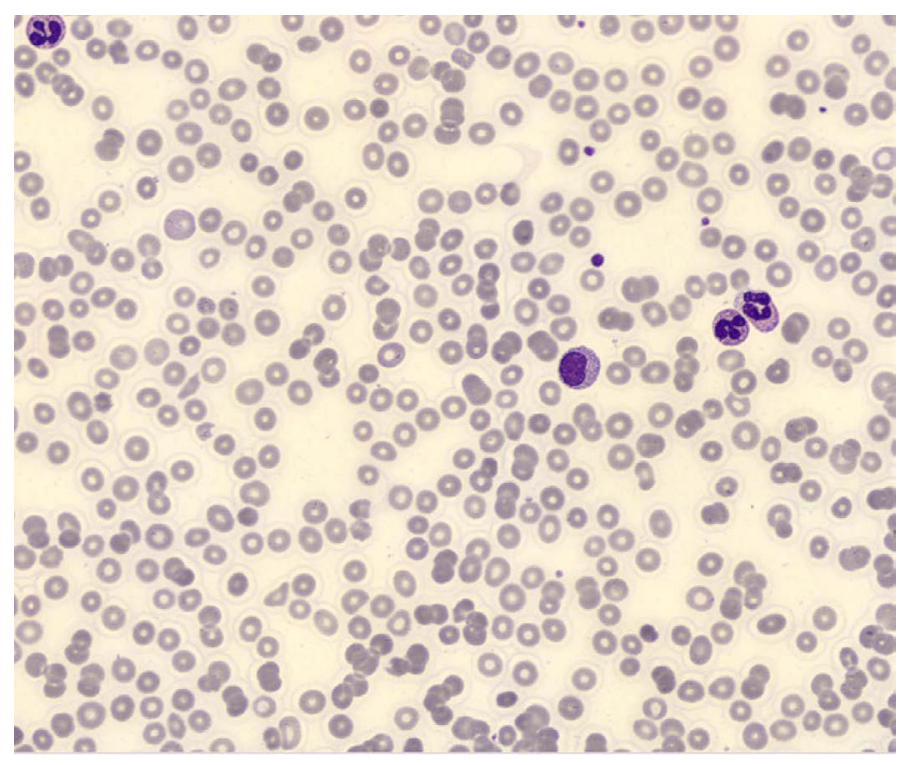

Figure 2. Peripheral blood smear (Hematoxilin-Eosin) 7 days after liver transplantation, showing complete resolution of the clinical picture poorly understood (Figure 2). Finally, our case report shows for the first time a very rapid reversion of SCA after LT with prompt decline of hemolysis indexes already within 24 hours. We speculated that this very rapid regression could be also used as an early marker of graft function but further investigations are required.

\section{References}

1. Alexopoulou A, Vasilieva L, Kanellopoulou T, Pouriki S, Soultati A, et al. (2014) Presence of spur cells as a highly predictive factor of mortality in patients with cirrhosis. J Gastroenterol Hepatol 29: 830-834.

2. Allen Smith J, Lonergan ET, Sterling K (1964) Spur cells anemia - hemolytic anemia with red cells resembling acanthocytes in alcoholic cirrhosis. NEJM 271: 396-398.

3. Vassiliadis T, Mpoumponaris A, Vakalopoulou S, Giouleme O, Gkissakis D, et al (2010) Spur cells and spur cell anemia in hospitalized patients with advanced liver disease: Incidence and correlation with disease severity and survival. Hepatol Res 40: $161-170$

4. Sousa JM, Giraldez A, De Blas JM, Diaz C, Pareja F, et al. (2002) Spur cell anemia in hepatic cirrhosis: incidence, prognosis and reversibility after liver transplantation. Hepatol 36: 64.

5. Malik P, Bogetti D, Sileri P, Testa G, Blumenthal SA, et al. (2002) Spur-cell anaemia in alcoholic cirrhosis: cure by orthotopic liver transplantation and recurrence after liver graft failure. Int Surg 87: 201-204.

Copyright: $@ 2019$ Santopaolo F. This is an open-access article distributed under the terms of the Creative Commons Attribution License, which permits unrestricted use, distribution, and reproduction in any medium, provided the original author and source are credited. 